\title{
Dependence of solar cycles duration on the magnitude of the annual module of the sunspots magnetic field
}

\author{
Valery N. Krivodubskij and Natalia I. Lozitska \\ Astronomical Observatory of Kyiv National Taras Shevchenko University \\ Observatorna Street 3, Kyiv - 53, 04053, Ukraine \\ email: krivod1@observ.univ.kiev.ua \\ nloz@observ. univ.kiev.ua
}

\begin{abstract}
The dependence of the solar cycle duration, $T$, on the 3 years averaged module of the large-scale sunspots magnetic fields $(30-60 \operatorname{arcsec}), B_{s p}$ index, was investigated on the base of about 10,000 visual observations conducted during last eight (16-23) cycles. It was found that the duration $T$ of the investigated cycles linearly depends on the index $B_{s p}$ of the magnetic fields observed during 3 years on decline phase of the solar cycle (second, third and fourth years after solar maximum $\left.T_{\max }\right)$. Namely, the duration of the cycles $T$ was varied between 9,5 and 12,5 years, when the magnetic index $B_{s p}$ was ranged from 2450 to $2600 \mathrm{G}$. An explanation for this dependence is proposed within the framework of non-linear $\alpha \Omega$ - dynamo model. We found the following equation for the dependence of solar dynamo-period on magnetic index: $T \approx B_{s p}^{3 / 2}$. Therefore, the large observed index $B_{s p}$, the longer calculated period $T$.
\end{abstract}

Keywords. Sun: activity, Sun: sunspots, magnetic fields

The new index of annual averaged module of the magnetic fields for the large-scale sunspots with penumbra diameter 30-60 arcsec $(22-44 \mathrm{Mm}), B_{s p}$, measured by visual method on the Zeeman splitting in the Fe I $\lambda \lambda 525.02$ and $630.25 \mathrm{~nm}$ lines was proposed (Lozitska 2005). The dependence of duration of last eight (16-23) solar cycles, $T$, on the observed annual index, $B_{s p}$, was investigated by Lozitska this year. Firstly, it was ascertained the dependence of sunspot magnetic field value on the time, years of cycle relatively maximum epoch, $T_{\max }$, of the average solar period (Fig. 1, where $B$ corresponds to index $B_{s p}$ ). Then it was found that the duration of the investigated cycles, $T$, was varied between 9,5-12,5 years, when the maximal 3 years averaged magnitude of magnetic induction, $B_{(\operatorname{Tmax}+3)}$ (index $B_{s p}$ ), was ranged from 2450 to $2600 \mathrm{G}$ (Fig. 2).

An explanation for the derived dependence is proposed within the framework of nonlinear $\alpha \Omega$ - dynamo model. According to our conception (Krivodubskij 2012) the magnetic index $B_{s p}$ reflects information on values of the deep toroidal field $B_{T}$ in the solar convection zone (SCZ). So this index could be used for the dynamo-period estimation. In this case the period of solar dynamo-cycle in non-linear regime is determined by equation $T=2 \pi /\{(1 / 2)|\alpha(\beta) \partial \Omega / \partial r|\}^{1 / 2}$ where $\alpha$ is the parameter of mean helicity of turbulent convective pulsations, $\partial \Omega / \partial r$ is the radial gradient of angle velocity, $\Omega$, in the SCZ, $\alpha(\beta)=\alpha_{0} \Psi_{\alpha}(\beta)$ is the helicity parameter of the turbulent pulsations, $\alpha_{0}$ is the "non-magnetic" helicity parameter, $\beta=B_{s p} / B_{e q}$ is the normalized magnetic field, $B_{e q} \approx v(4 \pi \rho)^{1 / 2}$ is the equipartition magnetic induction, $v$ is the small-scale turbulent velocity, $\Psi_{\alpha}(\beta)$ is the quenching-function. We took into account that the alphaquenching for strong magnetic field $(\beta \gg 1)$ is expressed by the equation $\alpha(\beta)=\alpha_{0} \Psi_{\alpha}(\beta)$ $=\alpha_{0} 15 \pi / 64 \beta^{3}$ (Rüdiger \& Kitchatinov 1993; Krivodubskij 2005). Since the period of 


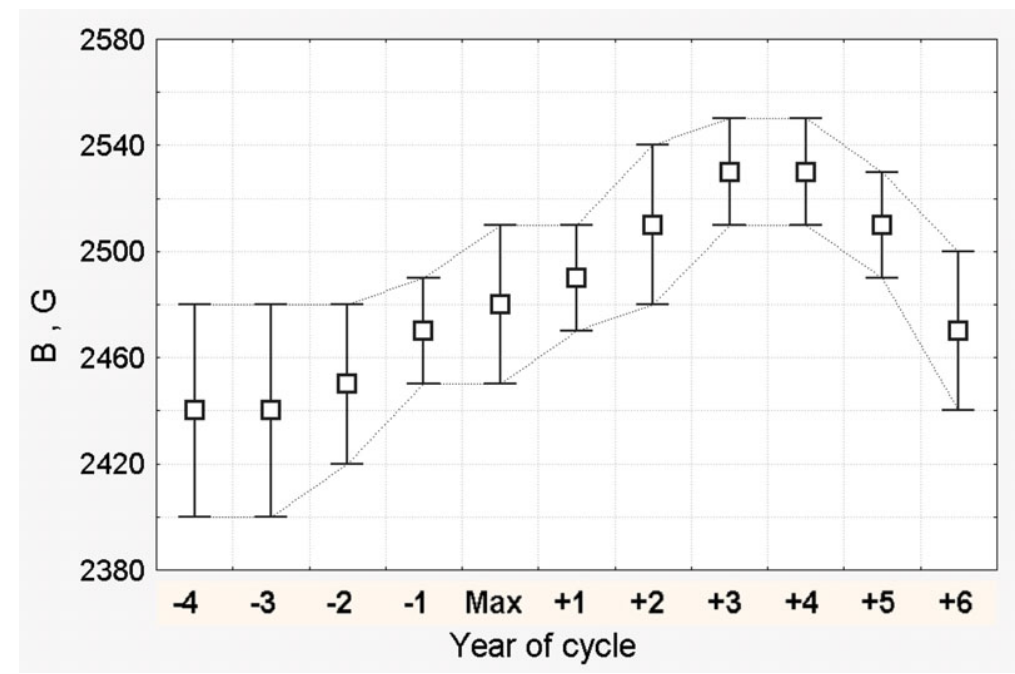

Figure 1. Sunspots magnetic field 3 year averaged, $B_{(\operatorname{Tmax}+3)}$, relatively solar maximum, $T_{\text {max }}$, against years of average cycle period ( $16-23$ cycles).

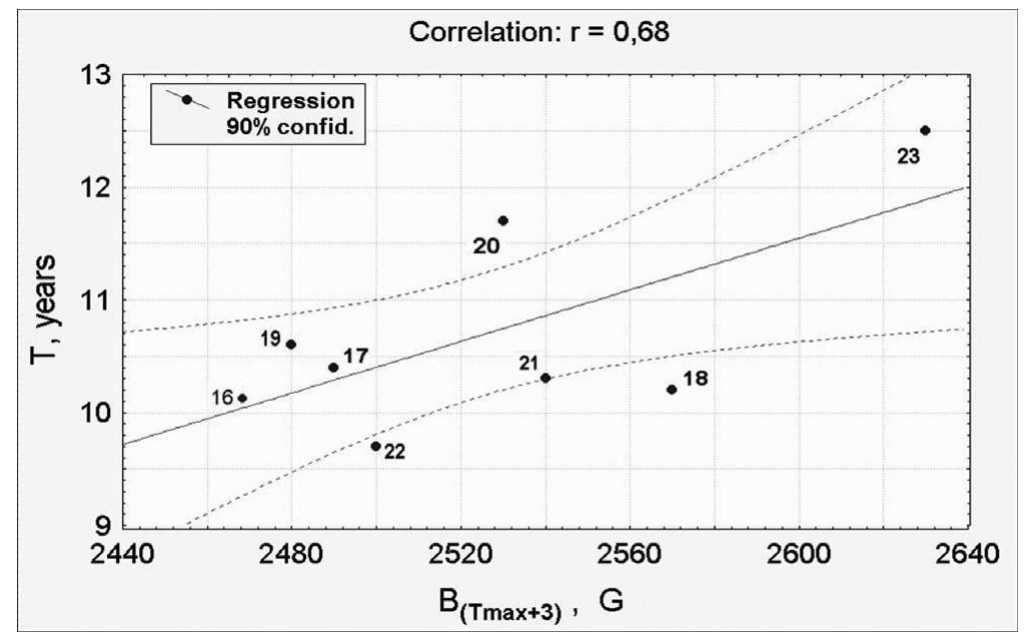

Figure 2. Solar cycles duration, $T$, dependence on 3 years average sunspots magnetic field, $B_{(\text {Tmax }+3)}$ (index $B_{s p}$ ), during 2, 3 and 4 -th years after epoch of maximum sunspots relatively numbers (16 -23 cycles).

dynamo-cycle $T$ is reversely proportional to square root from the parameter $\alpha(\beta)$ then we found following equation for the period dependence on the magnetic parameters, $T \approx \beta^{3 / 2}\left(\approx B_{s p}^{3 / 2}\right)$. Thereby, we received following correlation between magnetic index and cycle period: the large observed index $B_{s p}$, the less calculated quenching-function $\Psi_{\alpha}\left(B_{s p}\right)$, and therefore the longer calculated period $T$.

\section{References}

Krivodubskij, V. N. 2005, AN, 3, 61

Krivodubskij, V. N. 2012, This volume

Lozitska, N. I. 2005, Kinenatika i Fizika Nebes. Tel (Suppl.), 5, 151

Rüdiger, G. \& Kitchatinov, L. L. 1993, AA, 269, 581 